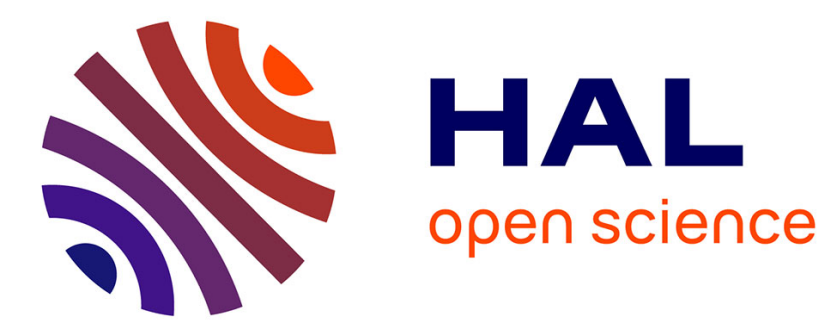

\title{
Back-projection inversion of a conical Radon transform
} Javier Cebeiro, M. Morvidone, M K K Nguyen

\section{To cite this version:}

Javier Cebeiro, M. Morvidone, M K K Nguyen. Back-projection inversion of a conical Radon transform. Inverse Problems in Science and Engineering, 2015, 24 (2), pp.1 - 25. 10.1080/17415977.2015.1034121 . hal-01260333

\section{HAL Id: hal-01260333 \\ https://hal.science/hal-01260333}

Submitted on 13 Apr 2018

HAL is a multi-disciplinary open access archive for the deposit and dissemination of scientific research documents, whether they are published or not. The documents may come from teaching and research institutions in France or abroad, or from public or private research centers.
L'archive ouverte pluridisciplinaire HAL, est destinée au dépôt et à la diffusion de documents scientifiques de niveau recherche, publiés ou non, émanant des établissements d'enseignement et de recherche français ou étrangers, des laboratoires publics ou privés. 
To appear in Inverse Problems in Science and Engineering

Vol. 00, No. 00, Month 20XX, 1-25

\title{
Back-projection Inversion of a Conical Radon Transform
}

\author{
J. Cebeiro ${ }^{\mathrm{a}, \mathrm{b}}$, M. Morvidone $\mathrm{a}^{\mathrm{a}, \mathrm{c} *}$ and M. K. Nguyen ${ }^{\mathrm{b}}$ \\ ${ }^{a}$ Centro de Matemática Aplicada, Universidad Nacional de San Martín, Av. 25 de Mayo y \\ Francia, B1650HMP, Gral. San Martín, Buenos Aires, Argentina; \\ ${ }^{b}$ Equipes de Traitement de l'Information et Systèmes (ETIS) ENSEA/University of \\ Cergy-Pontoise/CNRS UMR CNRS 8051, F-95014 Cergy-Pontoise Cedex, France; \\ ${ }^{c}$ Facultad Regional Buenos Aires, Universidad Tecnológica Nacional, Mozart 2300 (C 1407 \\ IVT), Buenos Aires, Argentina
}

(Received 00 Month 20XX; final version received 00 Month 20XX)

\begin{abstract}
In an effort to deal with many ionizing radiation imaging mechanisms involving the Compton effect, we study a Radon transform on circular cone surfaces having a fixed axis direction, which is called here conical Radon transform (CRT). Concretely we seek to recover a density function $f(x, y, z)$ in $\mathbb{R}^{3}$ from its integrals over such circular cone surfaces or its conical projections. Although the existence of the inverse CRT has been established, it is the aim of this work to use this result to extent the concept of back-projection well known in Computed Tomography (CT) to this type of cone surfaces. We discuss in some details the features of back-projection in relation to the corresponding conical Radon transform adjoint operator as well as the filters that arise naturally from the exact solution of the inversion problem. This intuitive approach is attractive, lends itself to efficient computational algorithms and may provide hints and guide for more general back-projection methods on other classes of cone surfaces, for example occurring in Compton camera imaging. Comprehensive numerical simulations results are presented and discussed to illustrate and validate this approach based on the concept of back-projection.
\end{abstract}

Keywords: Radon transforms; gamma-ray imaging; Compton scattering; conical Radon transform; filtered back-projection (FBP)

AMS Subject Classifications: 44A12; 65J22; 65R10; 92C55

\section{Introduction}

Emission imaging with ionizing radiation has made a quantum leap when it was proposed about four decades ago to use coincidence detection of two gamma ray events (a Compton scattering and an absorption) to improve sensitivity realizing the so-called electronic collimation. This concept can be implemented by removing the mechanical collimator of a gamma camera and by adding a scattering plane detector put in front of it. The new device is called a Compton camera [1,2], see Figure 1.

The idea is attractive but the corresponding mathematical challenge is huge. This is due to the fact that the detected signal, which is the amount of gamma photons of given energy arriving along the line joining the scattering site to the detection site contains the contribution of all the emitting sites of the radiating object situated on a circular cone sheet. One may speak of this data as a conical projection in analogy to the linear projection known in conventional emission (SPECT: Single Photon Emission Computed Tomography, or PET: Positron Emission Tomography) or absorption (CT) tomography

\footnotetext{
*Corresponding author. Email: mmorvidone@unsam.edu.ar
} 


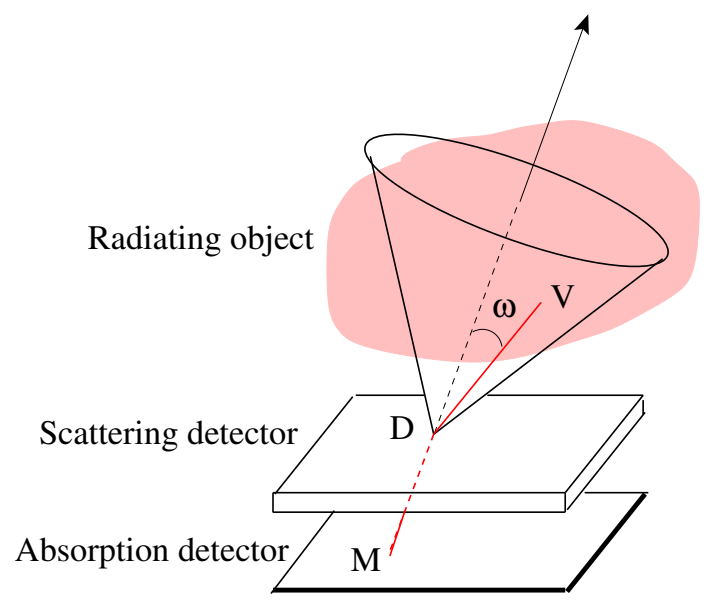

Figure 1. Compton Camera.

[3]. Such conical projections lead naturally to the notion of Conical Radon transform since linear projections have led to the Classical Line Radon transform. Several attempts have been made to find the inversion formula for this case [4-7].

However, many classes of conical projections can be found in $\mathbb{R}^{3}$. The ones relevant to one type of Compton cameras are conical projections that swing around a fixed point such that the cone vertex moves on a two-dimensional surface. The related Conical Radon transform has unfortunately no inversion formula to this date. To gain insight to the inversion of such a difficult problem, it is reasonable to consider first more tractable families of conical projections. This is why, in this work, we consider conical projections that have fixed axis direction. This family of conical projections has some nice symmetry properties, such as translational invariance in a plane perpendicular to the cone axis direction, which permit to find an inversion formula. The corresponding Conical Radon Transform (CRT) has been studied first by Cree and Bones although they did not succeed to get an inversion formula in [8]. It turns out that this CRT may be viewed as the working support of a particular Compton camera, which consists of the usual gamma camera with a hole collimator to which a scattering detector is placed ahead of it, see Figure 2. Of course technologically, this proposal is awkward since the presence of the mechanical collimator dispels the very concept of electronic collimation. Nonetheless, on a theoretical level this camera makes use of a class of special conical projections (with cone axis direction perpendicular to a plane), which belongs to the CRT.

In 2005, a first inversion formula for the CRT was established [9] in $\mathbb{R}^{3}$, using circular harmonic components of functions. Very recently, in 2014, under more general conditions Haltmeier [10] has obtained the general analytic inverse of a large class of CRT parameterized by two variables $(p, d)$, where $d$ is the space dimension and $p$ may be related to some physical effect. Thus this work opens a wide perspective in mathematics as well as potential applications in imaging ${ }^{1}$. In this paper, the aim is to develop efficient computation image reconstruction algorithms for the CRT, which positively illustrate these recent analytic results and possibly usher research into a new direction for emission imaging based on Radon transforms on cone surfaces.

To this end, we first revisit the concept of back-projection, well-known for the classical Radon transform in $\mathbb{R}^{2}$ and extend this notion to circular cone surfaces having fixed axis direction. We believe that this step may provide useful hints for constructing back-projections of more general conical projections occurring for example in Compton

\footnotetext{
${ }^{1}$ In two dimensions applications to emission imaging have been considered in [11]
} 


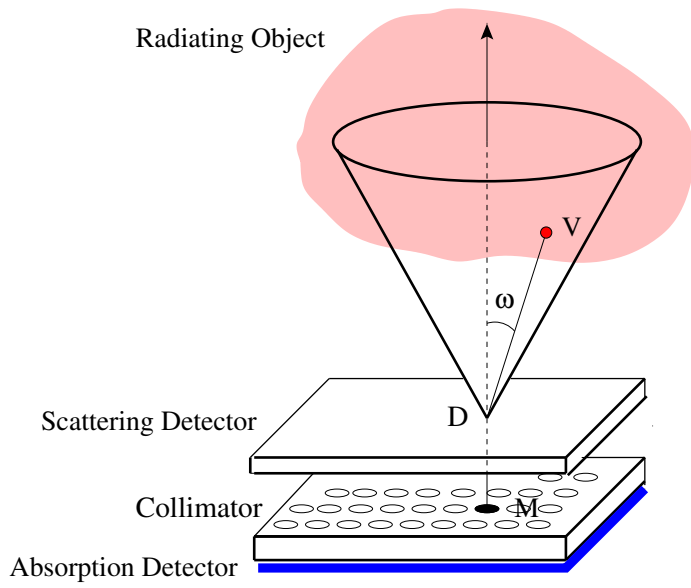

Figure 2. Cree-Bones Camera.

cameras, which is thought to be a future potential imaging tool for astronomy, industry applications, homeland security and medical diagnosis. Due to the inherent geometrical complexity of Compton camera data and in the absence of exact inversion formulas image reconstruction of distributed sources may be attempted first by back-projection method and later by adequate filtered back-projection. In new Compton cameras built on high pressure gaseous scintillation chamber, the conical projections may depend on more than three parameters and image reconstruction is performed mostly via back-projections [12]. We next show that this back-projection operation is globally represented by the adjoint of the CRT. Following the approach detailed in $[13,14]$, for the convenience of the reader, an inversion formula, which is a special case of the general results given in [10], is derived and shown to appear in the form of a filtered back-projection procedure. This speaks favorably for the design of efficient reconstruction algorithms as will be shown later.

The plan of this article is as follows. Section 2 introduces the CRT as resulting from the working of the Cree-Bones camera. It gives the definition of the CRT, its Point Spread Function and derives its analytic inversion formula. The next Section 3 is the main section on the notion of back-projection and how it is formulated for the CRT via an adjoint CRT as well as the occurrence of filters in the exact reconstruction formula (see Section 4). Then numerical simulation results are presented and commented in the following section. From a practical stand point, they are the necessary evidence which prove the relevance and importance of theoretical results since practitioners rely on them to build scanner prototypes. A conclusion closes the paper exposing some future research perspectives based on the present work.

\section{Image formation and the Conical Radon transform}

\subsection{Definition}

This section describes the working context needed for later use. Some of the results have appeared in the literature. The setup has been given in [9] for a slightly different model in which no account of radiation spreading is made. The inversion results as well as the expression of the Point Spread Function have been also obtained for this model although through the method of harmonic circular components. Inversion results for more generalized models are given in [10].

Consider the working of the Cree-Bones camera shown by Figure 2. A radiating site $V$ of an object emits isotropically radiation of calibrated energy $E_{0}$. Some part of it hits 
the scattering detector at site $D$ and then enters the collimator along its axis direction which is perpendicular to the scattering detector, so that the scattering angle is $\omega$. The absorption detector, which stands behind the scattering detector and after the collimator, is now set to register only scattered radiation of energy $E(\omega)$.

To write down the scattered flux density received by a site of the absorption detector, we need the total incoming flux density at site $D$, before scattering and the expression of the differential cross section of the Compton effect. This incoming flux density is due to the contribution of all emitting sites $V$ on the object that are located on a circular cone of vertex $D$, of axis perpendicular to the detectors and of opening angle $\omega$.

Let $f(x, y, z)$ be a nonnegative and smooth function in $\mathbb{R}^{3}$, representing the radioactivity density of the object, i.e. it is the density of radiation emitters in the object, which is in upper half-space $z>0$. In the coordinate system of Figure 3, [9], it can be seen that the integral of $f(x, y, z)$, called $\mathcal{R}_{\mathcal{C}} f\left(x_{D}, y_{D}, \omega\right)$ (the subindex $\mathcal{C}$ refers to the relevant cone and distinguish it from the classical Radon transform $\mathcal{R}$ ), on all the emitting sites $V$ on the cone, is given by

$$
\mathcal{R}_{\mathcal{C}} f\left(x_{D}, y_{D}, \omega\right)=\int_{0}^{\infty} \int_{0}^{2 \pi} d a f\left(x_{D}+r \sin \omega \cos \psi, y_{D}+r \sin \omega \sin \psi, r \cos \omega\right) \frac{1}{r^{2}}
$$

where $r=D V, d a=r \sin \omega d \psi d r$ (the integration measure on the cone) and $1 / r^{2}$ accounts for the photometric spreading of radiation from $V$ to $D$.

Equation (1) defines $\mathcal{R}_{\mathcal{C}} f\left(x_{D}, y_{D}, \omega\right)$ as the Conical Radon Transform (CRT) of $f(x, y, z)$. It is a function on $\mathcal{E}=\mathbb{R}^{2} \times[0, \pi / 2)$. From physical considerations, $f(x, y, z)$ is an integrable density on $\mathbb{R}^{2} \times \mathbb{R}^{+}$with compact support. Nevertheless, $\mathcal{R}_{\mathcal{C}} f\left(x_{D}, y_{D}, \omega\right)$ does not have compact support on $\mathcal{E}$. Recently Haltmeier in [10] has studied a more general CRT with parameters $(p, d)$, where $d$ is the space dimension and $p$ a variable describing the radial behavior due to possible physical effects. Most importantly he has established its analytic inverse. The case considered here corresponds to his CRT with $(p=2, d=3)$, which corresponds to an imaging system in real three dimensions in which radiation undergoes the usual photometric spreading law. Some properties of the CRT with $(p=0, d=3)$ are analyzed in $[9,15]$.

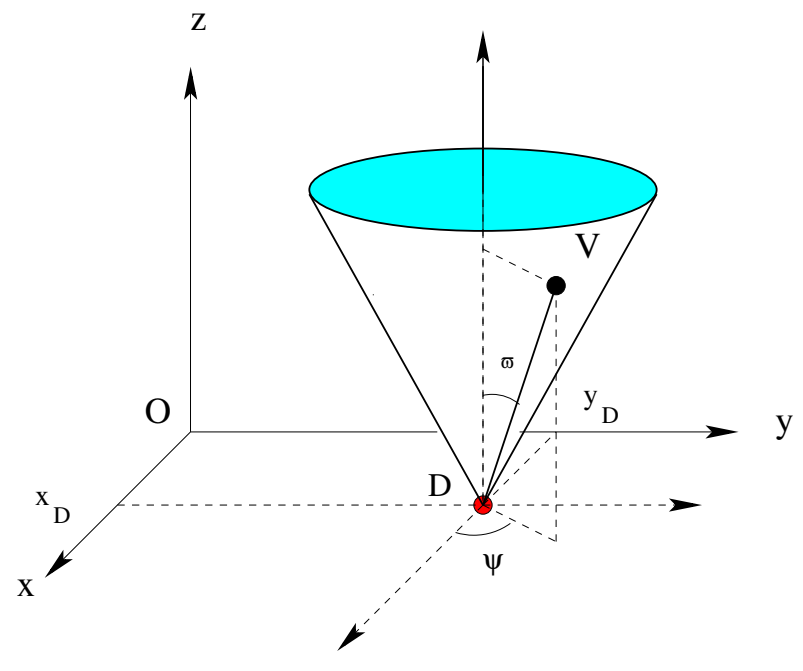

Figure 3. Used coordinate system, see [9]. 


\subsection{Image of a point source or Point Source Function (PSF)}

Proposition 2.1 The image of a unit point source is the kernel

$$
\mathcal{K}\left(x_{D}, y_{D}, \omega \mid x, y, z\right)=\frac{\cos \omega}{z^{2}} \delta\left(\sqrt{\left(x-x_{D}\right)^{2}+\left(y-y_{D}\right)^{2}}-z \tan \omega\right) .
$$

Proof. Equation (1) can be rewritten in terms of an integral on $z=r \cos \omega$

$$
\mathcal{R}_{\mathcal{C}} f\left(x_{D}, y_{D}, \omega\right)=\sin \omega \int_{0}^{\infty} \frac{d z}{z} \int_{0}^{2 \pi} d \psi f\left(x_{D}+z \tan \omega \cos \psi, y_{D}+z \tan \omega \sin \psi, z\right) .
$$

We set in equation $(3), f(x, y, z)=\delta\left(x-x_{0}\right) \delta\left(y-y_{0}\right) \delta\left(z-z_{0}\right)$ when the object is a unit point source at site $\left(x_{0}, y_{0}, z_{0}\right)$. Then $\mathcal{R}_{\mathcal{C}} f\left(x_{D}, y_{D}, \omega\right)=\mathcal{K}\left(x_{D}, y_{D}, \omega \mid x_{0}, y_{0}, z_{0}\right)$, the kernel of the CRT. Integration over $z$ yields

$\mathcal{K}\left(x_{D}, y_{D}, \omega \mid x_{0}, y_{0}, z_{0}\right)=\frac{\sin \omega}{z_{0}} \int_{0}^{2 \pi} d \psi \delta\left(x_{0}-x_{D}-z_{0} \tan \omega \cos \psi\right) \delta\left(y_{0}-y_{D}-z_{0} \tan \omega \sin \psi\right)$.

We convert the two-dimensional cartesian delta function into two-dimensional polar delta function according to

$$
\delta\left(\xi-\xi^{\prime}\right) \delta\left(\eta-\eta^{\prime}\right)=\frac{1}{\sqrt{\xi^{\prime 2}+\eta^{\prime 2}}} \delta\left(\sqrt{\xi^{\prime 2}+\eta^{\prime 2}}-\sqrt{\xi^{2}+\eta^{2}}\right) \delta\left(\theta-\theta^{\prime}\right)
$$

where $\theta=\arg \eta / \xi$ and $\theta^{\prime}=\arg \eta^{\prime} / \xi^{\prime}$.

To get the result we set

$$
\begin{aligned}
\xi & =x_{0}-x_{D}, \quad \xi^{\prime}=z_{0} \tan \omega \cos \psi \\
\eta & =y_{0}-y_{D}, \quad \eta^{\prime}=z_{0} \tan \omega \sin \psi .
\end{aligned}
$$

Then we obtain

$$
\begin{aligned}
\rho & =\sqrt{\left(x_{0}-x_{D}\right)^{2}+\left(y_{0}-y_{D}\right)^{2}} & \rho^{\prime} & =z_{0} \tan \omega \\
\theta & =\tan ^{-1} \frac{y_{0}-y_{D}}{x_{0}-x_{D}} & \theta^{\prime} & =\psi .
\end{aligned}
$$

Equation (4) becomes

$$
\begin{gathered}
\mathcal{K}\left(x_{D}, y_{D}, \omega \mid x_{0}, y_{0}, z_{0}\right)=\frac{\sin \omega}{z_{0}} \int_{0}^{2 \pi} d \psi \\
\frac{1}{\sqrt{\left(x_{0}-x_{D}\right)^{2}+\left(y_{0}-y_{D}\right)^{2}}} \delta\left(\sqrt{\left(x_{0}-x_{D}\right)^{2}+\left(y_{0}-y_{D}\right)^{2}}-z_{0} \tan \omega\right) \delta(\theta-\psi) .
\end{gathered}
$$

The $\psi$-integration yields 1 and equation (2) is obtained since $\sqrt{\left(x_{0}-x_{D}\right)^{2}+\left(y_{0}-y_{D}\right)^{2}}=z_{0} \tan \omega$.

In fact, the presence of the delta function means that the two sets of coordinates $\left(x_{0}, y_{0}, z_{0}\right)$ and $(x, y, z)$ are related by an homogeneous equation. Thus given the point source $\left(x_{0}, y_{0}, z_{0}\right)$, the variables in Radon space $\left(x_{D}, y_{D}, \omega\right)$ are linked the other by this 
homogeneous equation. For fixed $\omega$, we see that the point $D=\left(x_{D}, y_{D}, 0\right)$ is on a circle in the $x O y$ plane of center $(x, y, 0)$ and radius $z_{0} \tan \omega$.

\subsection{Inversion}

Let $\hat{f}$ be the Fourier transform on the first two variables of a function $f$, i.e.,

$$
\hat{f}(u, v, \cdot)=\int_{\mathbb{R}} \int_{\mathbb{R}} d x d y f(x, y, \cdot) e^{-2 i \pi(u x+v y)} .
$$

THEOREM 2.2 The function $f$ is reconstructed from the two-dimensional Fourier transform of the data $\mathcal{R}_{\mathcal{C}} f$ by the following integral

$$
\begin{gathered}
f(x, y, z)= \\
2 \pi z^{2} \int_{\mathbb{R}^{2}} d u d v e^{2 i \pi(x u+y v)}\left(u^{2}+v^{2}\right) \int_{0}^{\pi / 2} d \omega \frac{J_{0}\left(2 \pi \tan \omega \sqrt{\left(u^{2}+v^{2}\right)} z\right)}{\cos ^{3} \omega} \widehat{\mathcal{R}_{\mathcal{C}}} f(u, v, \omega) .
\end{gathered}
$$

where $J_{0}(\cdot)$ is the Bessel function of the first kind and order zero.

Proof. To get the inverse formula of the CRT, we take the Fourier transform of equation (1) first and rewrite it in polar coordinates in order to convert it to a Hankel transform of order zero, which is readily invertible.

We first apply the Fourier transform to both sides of equation (1). The left-hand-side becomes $\widehat{\mathcal{R}_{\mathcal{C}}} f(u, v, \omega)$. We get for the right-hand-side

$$
\begin{aligned}
= & \sin \omega \int_{0}^{2 \pi} d \psi \int_{0}^{\infty} \frac{d r}{r} \\
& \int_{\mathbb{R}} \int_{\mathbb{R}} d x_{D} d y_{D} f\left(x_{D}+r \sin \omega \cos \psi, y_{D}+r \sin \omega \sin \psi, r \cos \omega\right) e^{-2 i \pi\left(u x_{D}+v y_{D}\right)}, \\
= & \sin \omega \int_{0}^{2 \pi} d \psi \int_{0}^{\infty} \frac{d r}{r} \int_{\mathbb{R}} \int_{\mathbb{R}} d x d y f(x, y, r \cos \omega) e^{-2 i \pi(u(x-r \sin \omega \cos \psi)+v(y-r \sin \omega \sin \psi))}, \\
= & \sin \omega \int_{0}^{2 \pi} d \psi \int_{0}^{\infty} \frac{d r}{r} \hat{f}(u, v, r \cos \omega) e^{2 i \pi r \sin \omega(u \cos \psi+v \sin \psi)} .
\end{aligned}
$$

Now in Fourier space $(u, v)$, we change to polar coordinates via $u=q \cos \beta, v=q \sin \beta^{2}$. Equation (1) reads now

$$
\begin{aligned}
\widehat{\mathcal{R}_{\mathcal{C}}}(q, \beta, \omega) & =\sin \omega \int_{0}^{2 \pi} d \psi \int_{0}^{\infty} \frac{d r}{r} \hat{f}(q, \beta, r \cos \omega) e^{2 i \pi r \sin \omega(q \cos \beta \cos \psi+q \sin \beta \sin \psi)}, \\
& =2 \pi \sin \omega \int_{0}^{\infty} \frac{d r}{r} J_{0}(2 \pi q r \sin \omega) \hat{f}(q, \beta, r \cos \omega),
\end{aligned}
$$

\footnotetext{
${ }^{2}$ For the sake of readability, we keep the same name of the functions even after making a change of variables
} 
where $J_{0}(\cdot)$ is the Bessel function of order zero, given by the integral representation [16]

$$
J_{0}(x)=\frac{1}{2 \pi} \int_{0}^{2 \pi} d \theta e^{i x \sin \theta}
$$

Changing to variables $z=r \cos \omega$ and $t=\tan \omega$, for which $\sin \omega=\frac{t}{\sqrt{1+t^{2}}}$, and

$$
\frac{\sqrt{1+t^{2}}}{t} \widehat{\mathcal{R}_{\mathcal{C}}} f(q, \beta, \omega)=2 \pi \int_{0}^{\infty} \frac{d z}{z} J_{0}(2 \pi t z q) \hat{f}(q, \beta, z)
$$

This is essentially the Hankel transform of $\frac{\hat{f}(q, \beta, z)}{z^{2}}$. By applying the so-called Hankel identity [17], we obtain

$$
\hat{f}(q, \beta, z)=2 \pi q^{2} z^{2} \int_{0}^{\infty} t d t \frac{\sqrt{1+t^{2}}}{t} J_{0}(2 \pi t z q) \widehat{\mathcal{R}_{\mathcal{C}}} f(q, \beta, t)
$$

Finally, in order to recover $f$, we perform an inverse Fourier transform to this equation and get

$f(x, y, z)=2 \pi z^{2} \int_{0}^{\infty} d t \sqrt{1+t^{2}} \int_{0}^{\infty} d q q^{3} J_{0}(2 \pi t z q) \int_{0}^{2 \pi} d \beta e^{2 i \pi q(x \cos \beta+y \sin \beta)} \widehat{\mathcal{R}_{\mathcal{C}} f}(q, \beta, t)$.

Going back to variables $u, v, \omega$, for which $d t=d \omega / \cos ^{2} \omega, q=\sqrt{u^{2}+v^{2}}$ and $q d \beta d q=$ $d u d v$, we obtain the inversion formula of equation (5).

\section{Alternative inversion of the CRT by conical back-projection}

\subsection{A heuristic discussion on projections and back-projections}

As announced before, we wish to discuss in some details the notion of back-projection. In some outstanding future imaging concepts, image reconstruction usually faces the non-existence of analytic solutions. In this situation one may attempt a crude method of reconstruction, which goes under the name of back-projection. The purpose of this section is to analyze the very concept of back-projection in the widely known case of classical Radon transform and see how it may be understood in the case of the CRT. It is known that such an approach by back-projection is a very popular reconstruction method, albeit approximate, for many imaging integral transforms, such as the one in Compton Camera imaging.

\subsubsection{Back-projection for the classical Radon transform $\mathcal{R}$ [18]}

The notion of back-projection has its origin in the fact that the inverse formula of the classical Radon transform can be put under the form of the so-called filtered backprojection of the measured data, see for example [19]. The analytic reconstruction formula of the classical Radon transform for a function $f(x, y)$ may be put under the form of the integral over the rotation angle $\phi$ of a function which depends uniquely on the expression 
$(x \cos \phi+y \sin \phi)$, i.e.

$$
f(x, y)=\frac{1}{2 \pi^{2}} \int_{0}^{\pi} d \phi \int_{-\infty}^{\infty} d s \frac{1}{(x \cos \phi+y \sin \phi)-s} \frac{\partial}{\partial s} \mathcal{R} f(s, \phi) .
$$

Such a function, for given $\phi$ takes clearly a constant value on lines perpendicular to the direction specified by the angle $\phi$.

The word projection in Computed Tomography should not be confused with the notion of geometric projection in $\mathbb{R}^{2}$ of a point (or a figure) along a direction onto a line. Here it means the registered cumulated (or integrated) values of a physical density along a line in the plane at a point of this line. Generally the process of back-projecting this measured value means a kind of 'inversion', which assigns this measured value to all points on the measurement line. When this is done for all available projections in a given direction (specified by the angle $\phi$ ), a summation over all directions (which is sometimes called a 'summation image' in [19]) is carried out, a 'rough' image of the density is obtained. In fact this image suffers from artifacts and blurs. In some cases these reconstruction defects can be removed by using appropriate filters. This is why this method is favored as a first step in many imaging processes which do not have an analytic reconstruction formula.

Let $\mathcal{R} f(s, \phi)$ be the projection of some object density function $f(x, y)$ along the straight line $\mathcal{L}(s, \phi)$, where $s$ is the distance of the coordinate origin $O$ to this line and $\phi$ is the angle of the unit normal vector of the line with the axis $O x$. Assigning the value $\mathcal{R} f(s, \phi)$ to all points of the line $\mathcal{L}(s, \phi)$, means to construct from $\mathcal{R} f(s, \phi)$ a function of $(x, y)$, which takes this value for all $(x, y) \in \mathcal{L}(s, \phi)$. To achieve this goal, we use the canonical equation of the line $\mathcal{L}(s, \phi)$

$$
x \cos \phi+y \sin \phi-s=0
$$

and construct the function $\mathcal{R} f(x \cos \phi+y \sin \phi, \phi)$, by putting $s=x \cos \phi+y \sin \phi$ in $\mathcal{R} f(s, \phi)$. It can be verified that this function satisfies all the wanted requirements, hence the mapping

$$
\mathcal{R} f(s, \phi) \rightarrow \mathcal{R} f(x \cos \phi+y \sin \phi, \phi)
$$

represents the back-projection of $\mathcal{R} f(s, \phi)$ on $\mathcal{L}(s, \phi)$.

Conversely given a point $(x, y) \in \mathbb{R}^{2}$ and a specified value of $\phi$, there is only one projection line on which $(x, y)$ is situated. Consequently for this point $(x, y)$ and for given $\phi$, there corresponds only one projection $\mathcal{R} f(s, \phi)$, where $s$ is the distance of the origin to the projection line. If we put together all back-projections by summing on $\phi$, but keeping $(x, y)$ fixed, we get the 'summation image' (or the 'rough' reconstruction) of the density at $(x, y)$,

$$
\int_{0}^{\pi} d \phi \mathcal{R} f(x \cos \phi+y \sin \phi, \phi)
$$

\subsubsection{Back-projection for the Conical Radon transform or $\mathcal{R}_{\mathcal{C}}$}

As stated in the introduction, a conical projection means the integral of a density $f(x, y, z)$ on the upper sheet of a circular cone with vertical axis (parallel to $O z$ ), vertex 
at $\left(x_{D}, y_{D}\right)$ in the $x O y$-plane and opening angle $\omega$. So the measured quantity is the data $\mathcal{R}_{\mathcal{C}} f\left(x_{D}, y_{D}, \omega\right)$, a function of three variables in $\mathcal{E}=\mathbb{R}^{2} \times[0, \pi / 2)$.

Back-projecting this measured value $\mathcal{R}_{\mathcal{C}} f\left(x_{D}, y_{D}, \omega\right)$ on the measurement cone consists in constructing a function of $(x, y, z)$ from this data $\mathcal{R}_{\mathcal{C}} f\left(x_{D}, y_{D}, \omega\right)$, such that, when the running point $(x, y, z)$ sits on the cone $\mathcal{C}$, or $(x, y, z) \in \mathcal{C}$, this function takes the value $\mathcal{R}_{\mathcal{C}} f\left(x_{D}, y_{D}, \omega\right)$. For this construction, we use the parametric equations of the cone $\mathcal{C}$

$$
\begin{aligned}
& x=x_{D}+z \tan \omega \cos \psi, \\
& y=y_{D}+z \tan \omega \sin \psi, \\
& z=z
\end{aligned}
$$

where $\psi$ is given by

$$
\tan \psi=\frac{y-y_{D}}{x-x_{D}}
$$

and, similarly to equation (9), the mapping

$$
\begin{aligned}
\mathcal{R}_{\mathcal{C}} f\left(x_{D}, y_{D}, \omega\right) & \rightarrow \mathcal{R}_{\mathcal{C}} f(x-z \tan \omega \cos \psi, y-z \tan \omega \sin \psi, \omega) \\
& =\mathcal{R}_{\mathcal{C}} f\left(x-z \tan \omega \cos \tan ^{-1} \frac{y-y_{D}}{x-x_{D}}, y-z \tan \omega \sin \tan ^{-1} \frac{y-y_{D}}{x-x_{D}}, \omega\right),
\end{aligned}
$$

defines the back-projection of $\mathcal{R}_{\mathcal{C}} f\left(x_{D}, y_{D}, \omega\right)$ on the cone $\mathcal{C}$. It can be verified that this function satisfies the requirement that it takes the value $\mathcal{R}_{\mathcal{C}} f\left(x_{D}, y_{D}, \omega\right)$ at any point on the upper sheet of the cone $\mathcal{C}$.

Conversely given a point $(x, y, z) \in \mathbb{R}^{3}$ and a fixed value of $\omega$, this point may be found on an infinite number of circular cones of vertical axis, of opening angle $\omega$ and of vertex $\left(x_{D}, y_{D}, 0\right)$, which is on a circle $\Gamma$ of center $(x, y, 0)$ in the $x O y$-plane and of radius $\rho=\sqrt{\left(x_{D}-x\right)^{2}+\left(y_{D}-y\right)^{2}}=z \tan \omega$, see Figure 4 . The position of the vertex $\left(x_{D}, y_{D}, 0\right)$ on this circle is given by the angle $\psi$. Therefore the point $(x, y, z) \in \mathbb{R}^{3}$ belongs to an infinite set of conical projections parameterized by $(\psi, \omega) \in[0,2 \pi] \times[0, \pi / 2)$.

Hence in the back-projection process, with the aim to reconstruct the density $f(x, y, z)$ from the data $\mathcal{R}_{C} f\left(x_{D}, y_{D}, \omega\right)$, one should take into account this full infinite set of conical projections by an integration over $\psi \in[0,2 \pi]$

$$
\int_{0}^{2 \pi} d \psi \mathcal{R}_{\mathcal{C}} f(x-z \tan \omega \cos \psi, y-z \tan \omega \sin \psi, \omega)
$$

Then a second integration over $\omega \in[0, \pi / 2)$, would yield the 'summation image', which constitutes a 'rough image' reconstruction of the density $f(x, y, z)$, i.e.

$$
\int_{0}^{\pi / 2} d \omega \int_{0}^{2 \pi} d \psi \mathcal{R}_{\mathcal{C}} f(x-z \tan \omega \cos \psi, y-z \tan \omega \sin \psi, \omega)
$$




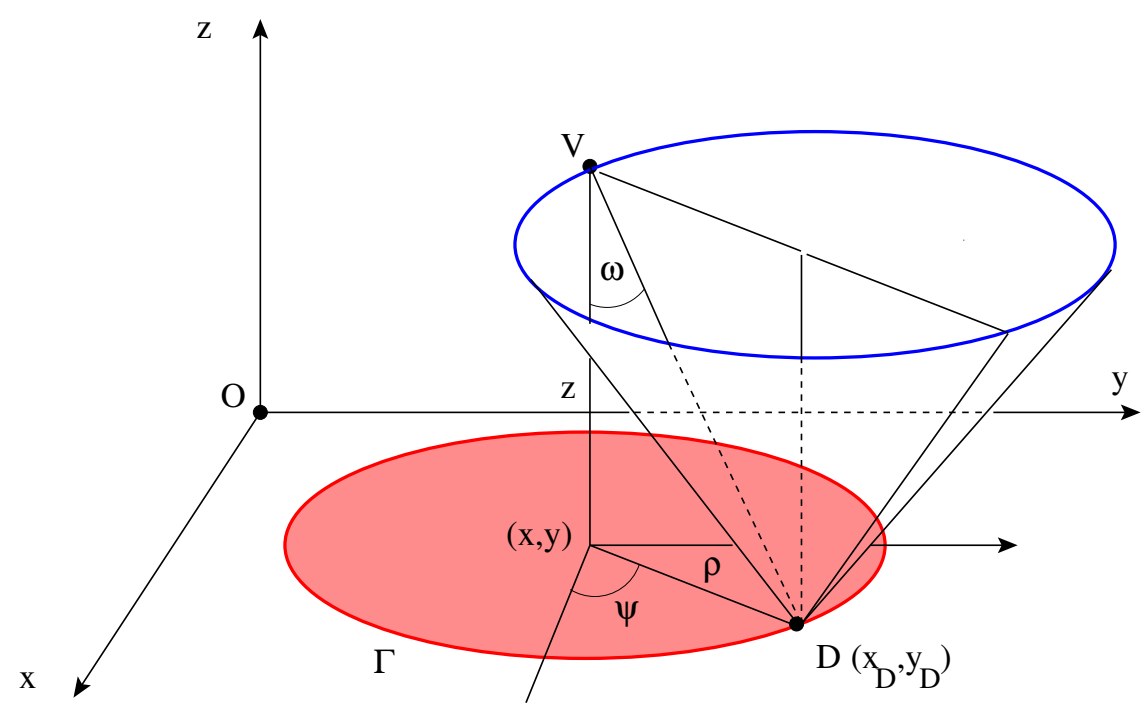

Figure 4. Point source and circle $\Gamma$

\subsection{The adjoint operator}

In this section we discuss the role of the adjoint operator (for the classical Radon transform as well as the conical Radon transform) in the back-projection operation. Broadly speaking the adjoint operator of a linear operator is its transpose. It is related to the dual of the same operator but a Hilbert structure on the space on which it acts must be specified ${ }^{3}$. In the present context, the linear operators being given by their real delta function kernels (such as (2)), we shall see how the adjoint operates on functions in image spaces. First we review the case of the classical Radon transform before going to the case of the conical Radon transform.

\subsubsection{The classical Radon transform adjoint}

As is well known the Radon transform maps functions in $\mathbb{R}^{2}$ to functions in $\mathbb{E}=\mathbb{R} \times[0, \pi]$, via

$$
\mathcal{R} f(s, \phi)=\int_{\mathbb{R}^{2}} d x d y \delta(x \cos \phi+y \sin \phi-s) f(x, y) .
$$

The adjoint $\mathcal{R}^{\dagger}$ is thus the mapping from functions in $\mathbb{E}=\mathbb{R} \times[0, \pi]$ to functions in $\mathbb{R}^{2}$, via

$$
\mathcal{R}^{\dagger} g(x, y)=\int_{\mathbb{R} \times[0, \pi]} d s d \phi \delta(x \cos \phi+y \sin \phi-s) g(s, \phi)
$$

Integration on $s$ yields directly the result of the back-projection described in the previous subsection

$$
\mathcal{R}^{\dagger} g(x, y)=\int_{0}^{\pi} d \phi g(x \cos \phi+y \sin \phi, \phi)
$$

\footnotetext{
${ }^{3}$ The difference between adjoint and dual is discussed in [20]
} 
So the intuitive notion of back-projection has a natural mathematical realization as the adjoint operator. More details on $\mathcal{R}^{\dagger}$ can be found in [18].

Suppose now that we take for $\mathcal{R} f(s, \phi)$ the classical Radon data for a point source at $\left(x_{0}, y_{0}\right)$ (or PSF), i.e.

$$
\mathcal{R} f(s, \phi)=\delta\left(x_{0} \cos \phi+y_{0} \sin \phi-s\right) .
$$

It will be back-projected on the line $\mathcal{L}(s, \phi)$ as $g(x \cos \phi+y \sin \phi, \phi)=\delta\left(\left(x-x_{0}\right) \cos \phi+\right.$ $\left.\left(y-y_{0}\right) \sin \phi\right)$. The resulting 'summation image', obtained by applying the adjoint Radon transform $\mathcal{R}^{\dagger}$, is not a delta function, as expected, but the following function (see [19])

$$
\int_{0}^{\pi} d \phi \delta\left(\left(x-x_{0}\right) \cos \phi+\left(y-y_{0}\right) \sin \phi\right)=\frac{1}{\sqrt{\left(x-x_{0}\right)^{2}+\left(y-y_{0}\right)^{2}}}
$$

which is clearly a 'rough' image of the delta function at $\left(x_{0}, y_{0}\right)$.

\subsubsection{The conical Radon transform adjoint}

Let $\mathcal{R}_{C}^{\dagger}$ be the adjoint of $\mathcal{R}_{C}$.

Proposition 3.1 The action of $\mathcal{R}_{C}^{\dagger}$ on a function $g\left(x_{D}, y_{D}, \omega\right)$ can be readily computed using the integral kernel of $\mathcal{R}_{\mathcal{C}}$ as

$$
\mathcal{R}_{C}^{\dagger} g(x, y, z)=\frac{1}{z} \int_{0}^{\pi / 2} d \omega \sin \omega \int_{0}^{2 \pi} d \psi g(x-z \tan \omega \cos \psi, y-z \tan \omega \sin \psi, \omega) .
$$

Proof. By definition using the PSF kernel (equation (2)), we have

$$
\begin{gathered}
\mathcal{R}_{C}^{\dagger} g(x, y, z)= \\
\int_{\mathbb{R}^{2}} d x_{D} d y_{D} \int_{0}^{\pi / 2} d \omega \frac{\cos \omega}{z^{2}} \delta\left(\sqrt{\left(x_{D}-x\right)^{2}+\left(y_{D}-y\right)^{2}}-z \tan \omega\right) g\left(x_{D}, y_{D}, \omega\right) .
\end{gathered}
$$

To calculate this integral we go to the variable $t=\tan \omega$, which implies that $d \omega=$ $d t /\left(1+t^{2}\right)$. Then denoting $G\left(x_{D}, y_{D}, t\right)=g\left(x_{D}, y_{D}, \omega\right)$, equation (22) becomes

$$
\begin{gathered}
\mathcal{R}_{C}^{\dagger} g(x, y, z)= \\
\frac{1}{z^{3}} \int_{\mathbb{R}^{2}} d x_{D} d y_{D} \int_{0}^{\infty} d t \frac{1}{\left(1+t^{2}\right)^{3 / 2}} \delta\left(\frac{\sqrt{\left(x_{D}-x\right)^{2}+\left(y_{D}-y\right)^{2}}}{z}-t\right) G\left(x_{D}, y_{D}, t\right) .
\end{gathered}
$$

The $t$-integration can be easily carried out, the result is

$$
\mathcal{R}_{C}^{\dagger} g(x, y, z)=\frac{1}{z^{3}} \int_{\mathbb{R}^{2}} d x_{D} d y_{D} \frac{G\left(x_{D}, y_{D}, \frac{\sqrt{\left(x_{D}-x\right)^{2}+\left(y_{D}-y\right)^{2}}}{z}\right)}{\left(1+\frac{\left(x_{D}-x\right)^{2}+\left(y_{D}-y\right)^{2}}{z^{2}}\right)^{3 / 2}}
$$


The next step consists in setting polar coordinates in the plane with origin at $(x, y)$, see Figure 4, so that

$$
\begin{aligned}
x-x_{D} & =\rho \cos \psi \\
y-y_{D} & =\rho \sin \psi \\
d x_{D} d y_{D} & =\rho d \psi d \rho .
\end{aligned}
$$

Equation (24) now reads

$$
\mathcal{R}_{C}^{\dagger} g(x, y, z)=\frac{1}{z^{3}} \int_{0}^{\infty} d \rho \rho \int_{0}^{2 \pi} d \psi \frac{G(x-\rho \cos \psi, y-\rho \sin \psi, \rho / z)}{\left(1+\frac{\rho^{2}}{z^{2}}\right)^{3 / 2}}
$$

Figure 4 shows precisely that $\rho / z=\tan \omega=t$. This is a geometric relation which should not be confused with the $\omega$ used in equation (21) as an integration variable in the definition of $\mathcal{R}_{C}^{\dagger}$. Hence replacing $\rho$ by $z t$ in equation (26), we get the expected result after going back to $g\left(x_{D}, y_{D}, \omega\right)$ via $G\left(x_{D}, y_{D}, t\right)=g\left(x_{D}, y_{D}, \omega\right)$.

Remark 3.2 If $\mathcal{L}^{2}\left(\mathbb{R}^{2} \times \mathbb{R}^{+}\right)$and $\mathcal{L}^{2}(\mathcal{E})$ are real valued function spaces equipped with their respective inner products

$$
\begin{aligned}
& \langle f, g\rangle=\int_{0}^{\infty} d z \int_{-\infty}^{\infty} \int_{-\infty}^{\infty} d x d y f(x, y, z) g(x, y, z) \\
& {[f, g]=\int_{-\infty}^{\infty} \int_{-\infty}^{\infty} d x_{D} d y_{D} \int_{0}^{\pi / 2} d \omega f\left(x_{D}, y_{D}, \omega\right) g\left(x_{D}, y_{D}, \omega\right)}
\end{aligned}
$$

$\mathcal{R}^{\dagger}$ may be defined by $\left[\mathcal{R}_{\mathcal{C}} f, g\right]=\left\langle f, \mathcal{R}_{\mathcal{C}}^{\dagger} g\right\rangle$ for all $g \in \mathcal{L}^{2}(\mathcal{E})$ and all $f \in \mathcal{L}^{2}\left(\mathbb{R}^{2} \times \mathbb{R}^{+}\right)$.

Proof.

$$
\begin{aligned}
{\left[\mathcal{R}_{\mathcal{C}} f, g\right]=} & \int_{\mathbb{R}^{2}} d x_{D} d y_{D} \int_{0}^{\pi / 2} d \omega\left(\mathcal{R}_{\mathcal{C}} f\left(x_{D}, y_{D}, \omega\right)\right) g\left(x_{D}, y_{D}, \omega\right), \\
= & \int_{\mathbb{R}^{2}} d x_{D} d y_{D} \int_{0}^{\pi / 2} d \omega \sin \omega \\
& \int_{0}^{2 \pi} d \psi \int_{0}^{\infty} \frac{d r}{r} f\left(x_{D}+r \sin \omega \cos \psi, y_{D}+r \sin \omega \sin \psi, r \cos \omega\right) g\left(x_{D}, y_{D}, \omega\right) \\
= & \int_{0}^{\infty} \int_{\mathbb{R}} \int_{\mathbb{R}} d x d y \frac{d z}{z} f(x, y, z) \int_{0}^{\pi / 2} d \omega \sin \omega \\
& \int_{0}^{2 \pi} d \psi g(x-z \tan \omega \cos \psi, y-z \tan \omega \sin \psi, \omega) \\
= & \left\langle f, \mathcal{R}_{\mathcal{C}}^{\dagger} g\right\rangle,
\end{aligned}
$$


where we have performed the change of variables

$$
\left\{\begin{array}{l}
x=x_{D}+r \sin \omega \cos \psi \\
y=y_{D}+r \sin \omega \sin \psi \\
z=r \cos \omega .
\end{array}\right.
$$

So the action of the adjoint $\mathcal{R}_{\mathcal{C}}^{\dagger}$ on $g$ has the following expression

$$
\mathcal{R}_{\mathcal{C}}^{\dagger} g(x, y, z)=\frac{1}{z} \int_{0}^{\pi / 2} d \omega \sin \omega \int_{0}^{2 \pi} d \psi g(x-z \tan \omega \cos \psi, y-z \tan \omega \sin \psi, \omega) .
$$

Remark 3.3 It can be checked that the adjoint operator of the conical Radon transform has the kernel given in proposition 2.1.

Proof. From equation (29), for an arbitrary function $g\left(x_{D}, y_{D}, \omega\right)$, we have

$$
\mathcal{R}_{\mathcal{C}}^{\dagger} g(x, y, z)=\frac{1}{z} \int_{0}^{\pi / 2} d \omega \sin \omega \int_{0}^{2 \pi} d \psi g(x-z \tan \omega \cos \psi, y-z \tan \omega \sin \psi, \omega) .
$$

For a point source at $\left(x_{0}, y_{0}\right)$ with $\omega_{0}$, we have $g\left(x_{D}, y_{D}, \omega\right)=\delta\left(x_{D}-x_{0}\right) \delta\left(y_{D}-\right.$ $\left.y_{0}\right) \frac{1}{2 \pi} \delta\left(\omega-\omega_{0}\right)$. Thus $\mathcal{R}_{C}^{\dagger} g(x, y, z)$ is

$$
\begin{aligned}
& =\frac{1}{z} \int_{0}^{\pi / 2} d \omega \sin \omega \int_{0}^{2 \pi} d \psi \delta\left(x-z \tan \omega \cos \psi-x_{0}\right) \delta\left(y-z \tan \omega \sin \psi-y_{0}\right) \frac{1}{2 \pi} \delta\left(\omega-\omega_{0}\right), \\
& =\frac{\sin \omega_{0}}{2 \pi z} \int_{0}^{2 \pi} d \psi \delta\left(x-x_{0}-z \tan \omega_{0} \cos \psi\right) \delta\left(y-y_{0}-z \tan \omega_{0} \sin \psi\right), \\
& =\frac{\sin \omega_{0}}{2 \pi z} \int_{0}^{2 \pi} d \psi \int_{-\infty}^{\infty} d u e^{2 i \pi u\left(x-x_{0}-z \tan \omega_{0} \cos \psi\right)} \int_{-\infty}^{\infty} d v e^{2 i \pi v\left(y-y_{0}-z \tan \omega_{0} \sin \psi\right)}, \\
& =\frac{\sin \omega_{0}}{2 \pi z} \int_{0}^{2 \pi} d \psi \int_{0}^{\infty} q d q \int_{0}^{2 \pi} d \beta e^{2 i \pi q \cos \beta\left(\sigma \cos \gamma-z \tan \omega_{0} \cos \psi\right)} e^{2 i \pi q \sin \beta\left(\sigma \sin \gamma-z \tan \omega_{0} \sin \psi\right)}, \\
& =\frac{\sin \omega_{0}}{2 \pi z} \int_{0}^{2 \pi} d \beta \int_{0}^{\infty} q d q e^{2 i \pi(q \cos \beta \sigma \cos \gamma+q \sin \beta \sigma \sin \gamma)} 2 \pi J_{0}\left(2 \pi q z \tan \omega_{0}\right), \\
& =\frac{\sin \omega_{0}}{2 \pi z} 2 \pi \int_{0}^{\infty} q d q 2 \pi J_{0}(2 \pi q \sigma) J_{0}\left(2 \pi q z \tan \omega_{0}\right) \text {, } \\
& =\frac{\sin \omega_{0}}{z} \frac{\delta\left(\sigma-z \tan \omega_{0}\right)}{z \tan \omega_{0}}, \\
& =\frac{\cos \omega_{0}}{z^{2}} \delta\left(\sqrt{\left(x-x_{0}\right)^{2}+\left(y-y_{0}\right)^{2}}-z \tan \omega_{0}\right) \text {. }
\end{aligned}
$$

The integration steps are shortened here.

\section{Filters arising from exact inversion formulas}

As noticed, reconstruction by simple back-projection does not lead to 'clean' images. To eliminate imperfections filters are used. When exact analytic inversion formulas exists, 
one can show that some operations which precede the back-projection can actually be interpreted as natural filters in the reconstruction process. We shall discuss successively the cases of the classical Radon transform and the conical Radon transform. In general in the absence of exact inversion formulas, the filtering operation is of importance for numerical inversion, in particular in practical cases, see [21]. The question is how to design the proper filters for a given reconstruction problem.

\subsection{Case of the classical Radon transform $\mathcal{R}$}

As pointed out earlier, the exact inversion formula for the classical Radon transform, appears as the back-projection not on the Radon data $\mathcal{R} f(s, \phi)$ but a particular transform of the Radon data, i.e.

$$
\int_{0}^{\infty} d s \frac{1}{(x \cos \phi+y \sin \phi)-s} \frac{\partial}{\partial s} \mathcal{R} f(s, \phi) .
$$

This operation (Hilbert transform composed with $s$-derivative) may be viewed as a 'filtering' process, which removes the imperfections inherent to the back-projection procedure.

\subsection{Case of the conical Radon transform $\mathcal{R}_{\mathcal{C}}$}

Remark 4.1 Comparing the equations (15) and (21), expressing the back-projection operation and the adjoint operation, we observe a slight difference: in the adjoint conical Radon transform the integration on $\omega$ has a measure $\sin \omega / z$. One may already say that the adjoint action is a filtered back-projection with the filter $\sin \omega / z$.

Proposition 4.2 The action of the conical Radon transform adjoint $\mathcal{R}_{\mathcal{C}}^{\dagger}$ on the conical Radon transform data $\mathcal{R}_{C} f\left(x_{D}, y_{D}, \omega\right)$ is given by

$$
\begin{gathered}
\mathcal{R}_{\mathcal{C}}^{\dagger} \mathcal{R}_{C} f(x, y, z)= \\
\frac{2 \pi}{z} \int_{0}^{\pi / 2} d \omega \sin \omega \int_{\mathbb{R}^{2}} d u d v e^{2 i \pi(u x+v y)} J_{0}\left(2 \pi z \tan \omega \sqrt{u^{2}+v^{2}}\right) \widehat{\mathcal{R}_{C} f}(u, v, \omega) .
\end{gathered}
$$

Proof. Equation (21) allows to write

$$
\mathcal{R}_{\mathcal{C}}^{\dagger} \mathcal{R}_{C} f(x, y, z)=\frac{1}{z} \int_{0}^{\pi / 2} d \omega \sin \omega \int_{0}^{2 \pi} d \psi \mathcal{R}_{\mathcal{C}} f(x-z \tan \omega \cos \psi, y-z \tan \omega \sin \psi, \omega) .
$$

We now insert the Fourier representation of $\mathcal{R}_{C} f\left(x_{D}, y_{D}, \omega\right)$ with respect to the two first variables in the previous equation

$$
\mathcal{R}_{C} f\left(x_{D}, y_{D}, \omega\right)=\int_{\mathbb{R}^{2}} d u d v e^{2 i \pi\left(u x_{D}+v y_{D}\right)} \widehat{\mathcal{R}_{\mathcal{C}} f}(u, v, \omega) .
$$

Performing the $\psi$-integration, we obtain the Bessel function $J_{0}\left(2 \pi z \tan \omega \sqrt{u^{2}+v^{2}}\right)$ and the expected result.

We are now in a position to write the exact inversion formula (5) under the form of the action of the conical Radon transform adjoint on modified - or 'filtered' - conical Radon transform data $\mathcal{R}_{C} f\left(x_{D}, y_{D}, \omega\right)$. 
PROPOSITION 4.3 The exact inversion formula (5) may be rewritten as the conical Radon transform adjoint of a filtered conical Radon transform data in Fourier space with respect to the two first variables

$$
\frac{z}{\sin \omega} z^{2} \frac{u^{2}+v^{2}}{\cos ^{3} \omega} \widehat{\mathcal{R}_{\mathcal{C}} f}(u, v, \omega)
$$

The first filter is due to the structure of $\mathcal{R}_{\mathcal{C}}^{\dagger}$ and the second and third filter arise from the exact inversion procedure.

Proof. Just take formula (5) and rearrange it in the form of equation (32) and collect the remaining terms as filtered conical Radon transform data in the Fourier space of the two first variables. As the adjoint form is related to the back-projection form, we have obtained the filtered back-projection of the inversion formula.

\section{Numerical Simulation Results}

In this section we describe the numerical implementation of the forward CRT and the inversion of the CRT using the filtered back-projection algorithm. Basically, the problem consists in generating the data (i.e. projections of an object) and then applying the proposed inversion algorithm in order to obtain reconstructions of the original object. A 3D version of the Shepp-Logan brain phantom intended for medical imaging study is used as the function to be reconstructed. Voxels in the phantom range values in the interval [0,1]. Figure 5 illustrates representative slices of the phantom, for the sake of clarity only planes labeled by $z=6,9,12,15,18,20,23,26,29,32,35,38,41,44,46,49,52$ and 55 out of 64 planes are shown. Additionally, an error analysis is carried out in order to assess the quality of the results.

\subsection{Discretization description}

A three-dimensional medium of size $N \times N \times N$ is discretized as follows: $N=64$ (arbitrary length unit), $\Delta x=\Delta y=\Delta z=1,-32 \leq x \leq 31,-32 \leq y \leq 31$ and $0 \leq z \leq 63$. The original object function is $f(x, y, z)$. The detector array of size $N_{d} \times N_{d}$ was discretized using $N_{d}=160, \Delta x_{D}=\Delta y_{D}=1,-80 \leq x_{D} \leq 79,-80 \leq y_{D} \leq 79$. In this scheme the central point in the detector plane $\left(x_{D}, y_{D}\right)=(0,0)$ coincides with the origin of coordinates of the medium $(x, y, z)=(0,0,0)$.

Both, the direct and inverse problems involve integrals (equations (1) and (21)) that must be computed numerically. Summations corresponding to numerical integration are carried out using the following steps: $\Delta r=1.2$ and $\Delta \psi=0.01 \mathrm{rad}\left(\cong 0.57^{\circ}, N_{\psi}=628\right)$. When points in the summation grid do not fit those of the discrete medium, a linear interpolation method is used to calculate the values of the function at the new positions. Considering that the quality of reconstructions relies heavily on the data, care must be taken in order to provide an adequate basis for the inverse operation. The dimensions of the plane detector are important parameters. The support of the CRT defined by equation (1) is not bounded, but this property is obviously lost when discretizing. In the discrete version of the CRT, the far most point with non-zero value depends on both the discretization of the projection angle and the size of the object under study. In the scheme used, given that upper central points project to the external areas of the projection plane, a truncation at the borders of the plane would reduce dramatically the 

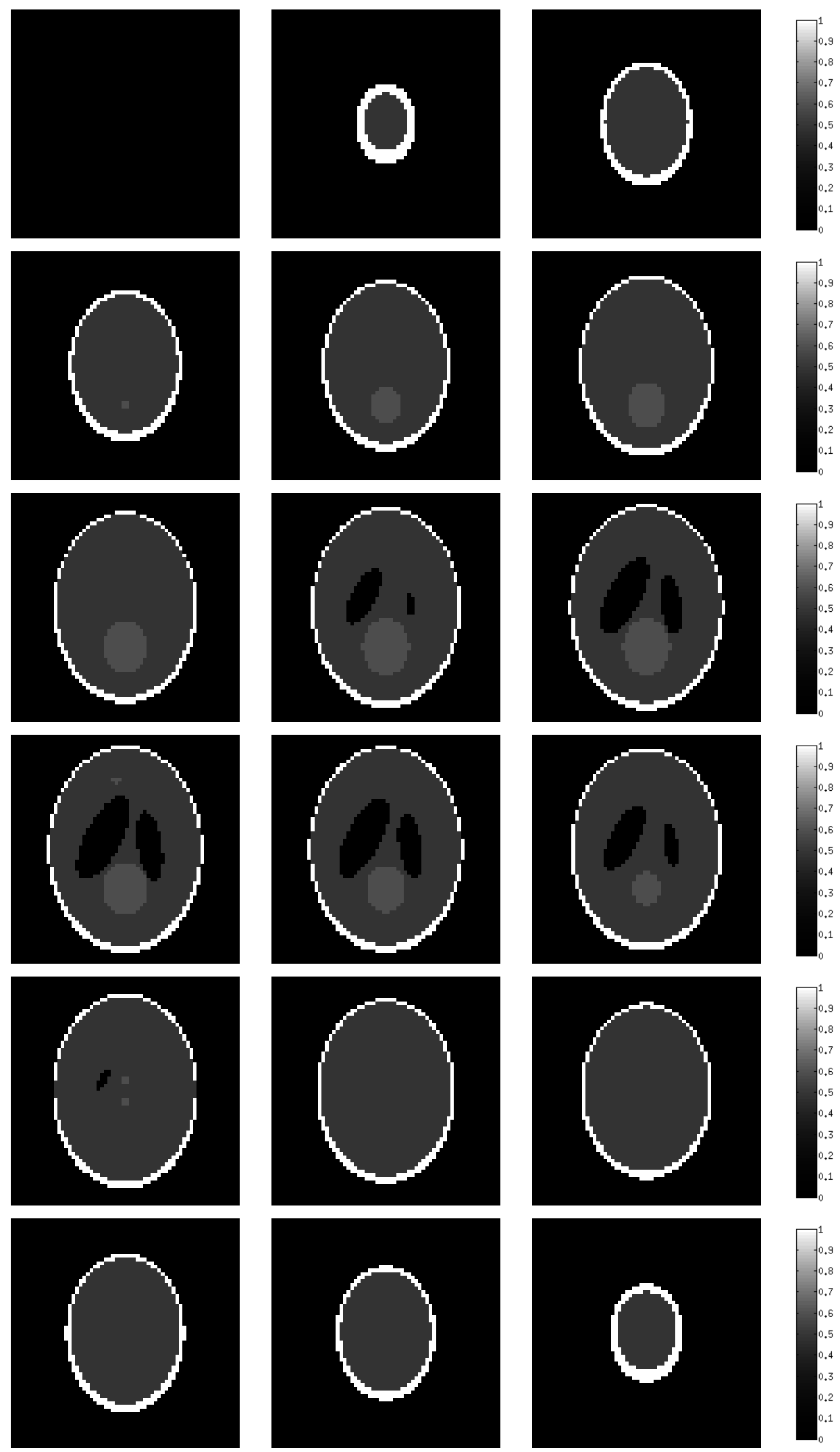

Figure 5. Original 3D Shepp-Logan phantom used for simulations. From top to bottom and from left to right planes with $z=6,9,12,15,18,20,23,26,29,32,35,38,41,44,46,49,52$ and 55 . 
quality of the upper planes of the reconstructions. In practice, this problem is addressed by using a square detector plane whose side is approximately three times larger than the support of the medium $\left(N_{d} \cong 3 N\right)$.

In order to reconstruct successfully a function of $N^{3}$ values, there is one requirement that must be fulfilled by the data. If there are $N^{3}$ voxels for which the value has to be determined, and since projections for a determined angle provide $N_{d}^{2}$ samples (detector array of $N_{d} \times N_{d}$ ), at least $\frac{N^{3}}{N_{d}^{2}}$ angles will be needed to acquire enough data to solve for the $N^{3}$ unknowns. In numerical inversions of the classical Radon transform, the reduced sampling density at the peripheral area of the plane is balanced by, at least, doubling this number [22]. Sixty four angles $\left(N_{\omega}=64\right)$ were considered in the range $\left[0, \frac{\pi}{2}\right)$ leading to an angular resolution of $\Delta \omega=0.0242 \mathrm{rad}\left(\cong 2.21^{\circ}\right)$.

\subsection{Projections}

Projections are calculated using equation (1) that describes the signal measured in a planar detector when it is exposed to scattered radiation under the hypothesis stated in Section 2.1. The discrete implementation of the direct CRT is straightforward and discretization parameters are discussed in Section 5.1. Figure 6 shows some images of the conical projections of the Shepp-Logan phantom labeled by its corresponding scattering angle. It can be seen that, for large angles, photons arrive at the borders of the projection plane leaving the central area deserted.

\subsection{Reconstruction algorithm by filtered back-projection}

In the proposed method, filtering is performed in the Fourier domain according to the formulas developed in Section 4.2. However, this process is not as straightforward as the direct problem is. Even though $\mathcal{R}_{\mathcal{C}}^{\dagger}$ applied to the inverse Fourier transform of (34) gives rise to an exact inversion formula of the CRT, its discrete implementation produces an artefactual result as a consequence of the divergence by the numerator of the third filter $\frac{u^{2}+v^{2}}{\cos ^{3} \omega}$. This behaviour, also known in the case of the ramp filter of the classical Radon transform, can be compensated by a convolution with a smoothing window using a technique known as apodization. This extra smoothing filter, intended exclusively for discrete reconstruction, is represented by $W(u, v)$ in the Fourier domain and operates together with the ones in equation (34). The global action of the four filters on the data is described by:

$$
\frac{z}{\sin \omega} z^{2} \frac{u^{2}+v^{2}}{\cos ^{3} \omega} W(u, v) \widehat{\mathcal{R}_{\mathcal{C}} f}(u, v, \omega)
$$

Figure 7 shows this implementation. The two-dimensional Fourier transform is applied to every slice of the projection $\mathcal{R}_{\mathcal{C}} f\left(x_{D}, y_{D}, \omega\right)$ labeled by the scattering angle $\omega$. The resulting planes, $\widehat{\mathcal{R}_{\mathcal{C}} f}(u, v, \omega)$, are multiplied by the corresponding four filters in equation (35). Finally the inverse Fourier transform is computed. The result is numerically integrated twice in order to obtain the reconstruction of $f(x, y, z)$ : first in $\psi$ (back-projection) and then in $\omega$ (summation of back-projections). The integration step $\Delta \psi=0.01 \mathrm{rad}$ is chosen as small as possible in order to reduce the artifacts produced by the discrete summation when the algorithm of back-projection is applied. 


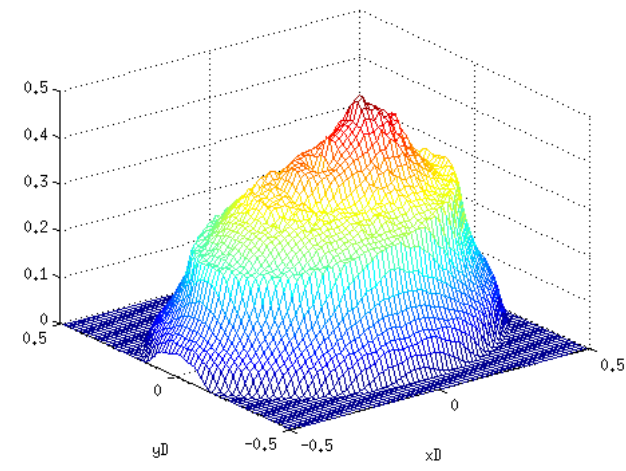

(a) $w=5^{\circ}$

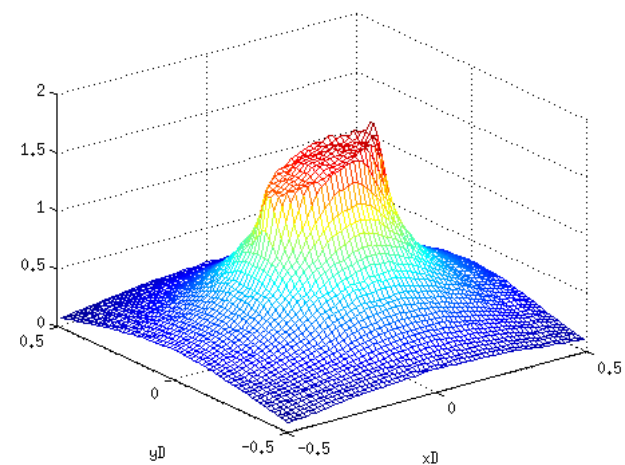

(c) $w=25^{\circ}$

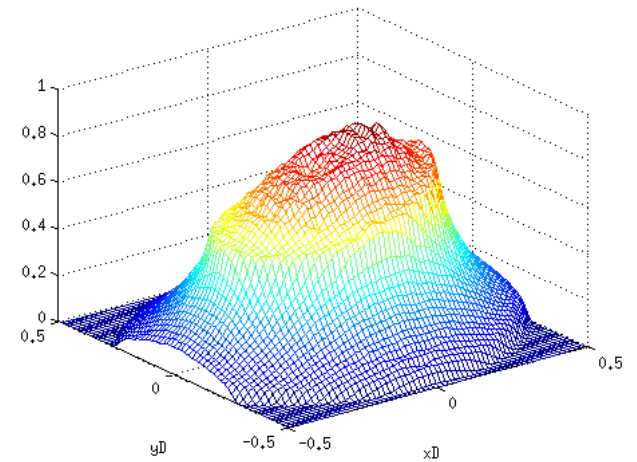

(b) $w=10^{\circ}$

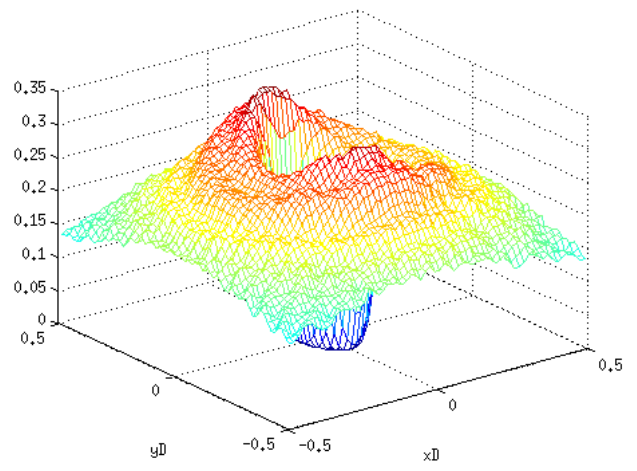

(d) $w=48^{\circ}$

Figure 6. Conical projections of Shepp-Logan phantom at four different scattering angles $\omega$.

$$
f(x, y, z)
$$

Eq. 1
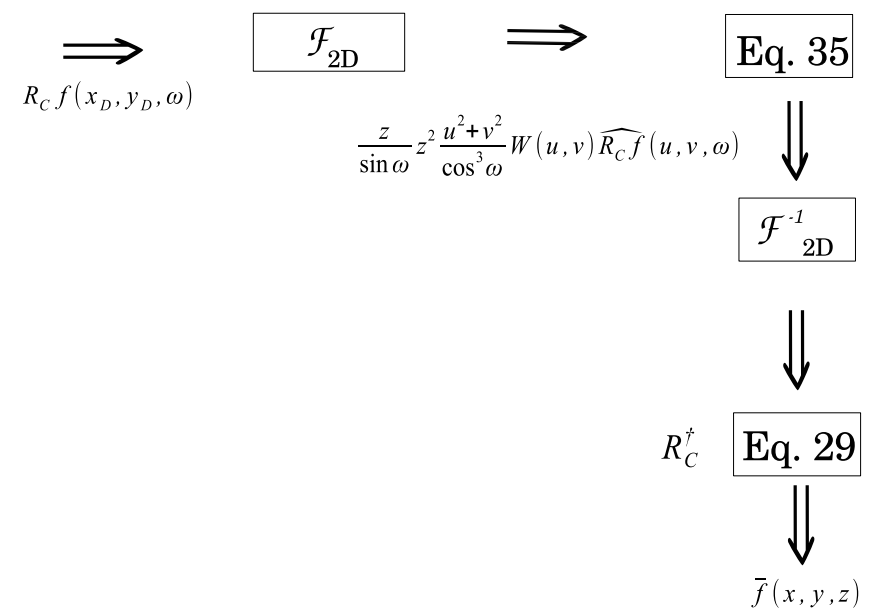

Figure 7. Scheme of reconstruction. 


\section{4. $\quad$ Smoothing windows}

There are several one-dimensional windows widely used for filtering in the context of the classical Radon transform: Shepp-Logan, Hamming, von Hann, cosine filters, etc. Here, we define three windows to act as $2 \mathrm{D}$ filters in a $3 \mathrm{D}$ reconstruction according to formula (35). These windows are defined as follows:

$$
\begin{gathered}
W_{H}(u, v)= \begin{cases}0.54+0.46 \cos \left(2 \pi \sqrt{u^{2}+v^{2}}\right), & \sqrt{u^{2}+v^{2}} \leq 0.5 \\
0.54, & \sqrt{u^{2}+v^{2}}>0.5\end{cases} \\
W_{S L}(u, v)= \begin{cases}\frac{\sin \left(2 \pi \sqrt{u^{2}+v^{2}}\right)}{2 \pi \sqrt{u^{2}+v^{2}}}, & \sqrt{u^{2}+v^{2}} \leq 0.5 \\
0, & \sqrt{u^{2}+v^{2}}>0.5\end{cases} \\
W_{\cos }(u, v)= \begin{cases}0.5\left(\cos \left(2 \pi \sqrt{u^{2}+v^{2}}\right)+1\right), & \sqrt{u^{2}+v^{2}} \leq 0.5 \\
0, & \sqrt{u^{2}+v^{2}}>0.5\end{cases}
\end{gathered}
$$

where $u$ and $v$ are the variables of the 2D Fourier domain, $(u, v) \in[-0.5,0.5] \times[-0.5,0.5]$. Figure 8 shows the product between the filter $\frac{u^{2}+v^{2}}{\cos ^{3} \omega}$, with $\omega$ fixed to 0 , and each of the smoothing windows. Given that they can be rewritten as functions of the radial variable $\rho=\sqrt{u^{2}+v^{2}}$, all of them exhibit circular symmetry. While Shepp-Logan and cosine windows reach zero values at high frequencies, Hamming window is never zero and, as a consequence, the harmonic content of the functions filtered using it is expected to be higher than the others.

\subsection{Image reconstruction quality}

In order to evaluate the quality of simulations we define two measures of the error NMSE (Normalised Mean Square Error) and NASE (Normalised Mean Absolute Error) [23].

$$
\begin{gathered}
\mathrm{NMSE}=\frac{100}{N^{3}} \frac{\sum_{i=0}^{N^{3}}\left(\mathbf{f}_{\mathbf{i}}-\tilde{\mathbf{f}}_{\mathbf{i}}\right)^{2}}{\max _{i}\left\{\mathbf{f}_{\mathbf{i}}\right\}} \\
\mathrm{NASE}=\frac{100}{N^{3}} \frac{\sum_{i=0}^{N^{3}}\left|\mathbf{f}_{\mathbf{i}}-\tilde{\mathbf{f}}_{\mathbf{i}}\right|}{\max _{i}\left\{\mathbf{f}_{\mathbf{i}}\right\}}
\end{gathered}
$$

where $\tilde{\mathbf{f}}$ and $\mathbf{f}$ are the reconstructed and reference functions respectively.

\subsection{Image reconstruction results}

Figure 9 shows the result of reconstructions using a cosine smoothing window. As in the case of the original phantom, only planes $z=6,9,12,15,18,20,23,26,29,32,35,38,41$, 44, 46, 49, 52 and 55 are exhibited. Table 1 shows NMSE and NASE for the smoothing windows proposed in Section 5.4. Even though the errors are slightly bigger in the case 


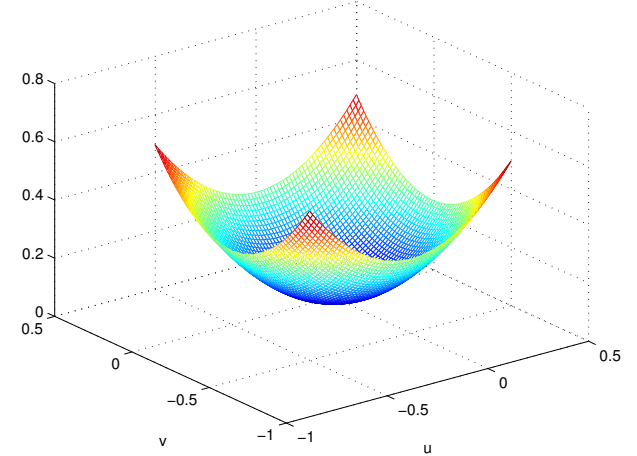

(a) Third filter

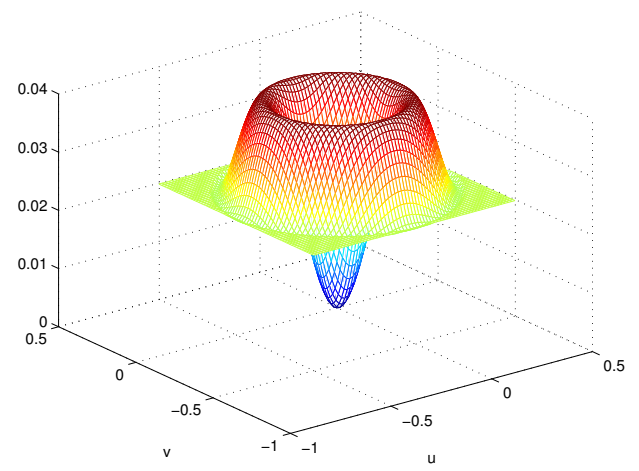

(c) Smoothing with Hamming window.

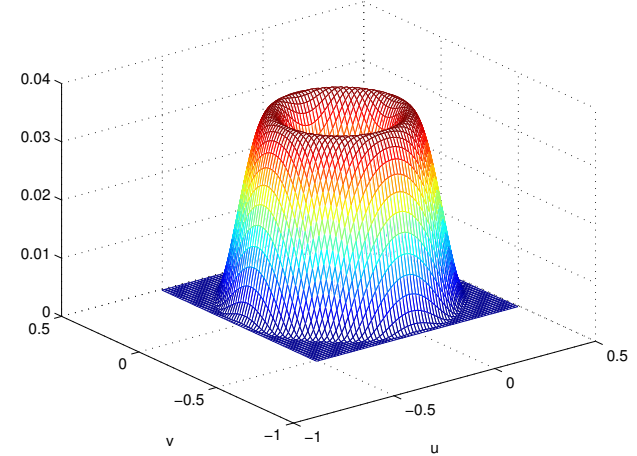

(b) Smoothing with cosine window.

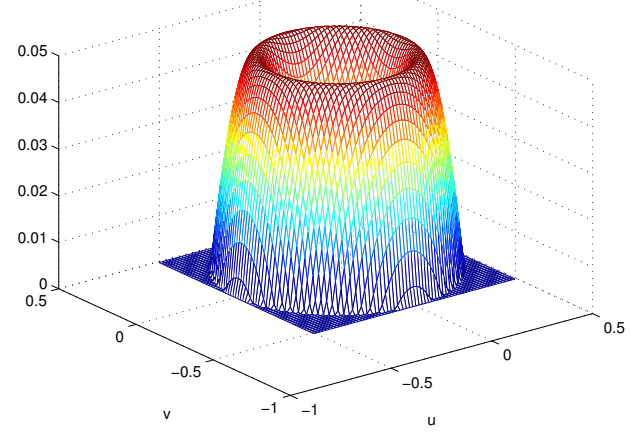

(d) Smoothing with Shepp-Logan window.

Figure 8. The filter $\frac{u^{2}+v^{2}}{\cos ^{3} \omega}(\omega=0)$ and its different smoothings.

Table 1. NMSE and NASE [\%] for different reconstructions from noiseless data.

\begin{tabular}{lccc}
\hline SNR & Shepp-Logan & Hamming & Cosine \\
\hline NMSE & 1.23 & 1.29 & 1.33 \\
NASE & 5.05 & 5.09 & 5.11 \\
\hline
\end{tabular}

of the cosine window, visually the reconstructions are similar with the three windows. Thus, we only show the results of the worst case which look already acceptable.

\subsection{Reconstructions from noisy projections}

The same procedure was applied to projections corrupted with additive gaussian noise with Signal to Noise Ratios SNR $=30.4 \mathrm{~dB}$ and SNR $=40.3 \mathrm{~dB}$. Measures of reconstruction errors were NMSE $=1.36 \%$ and NASE $=6.39 \%$ for $\mathrm{SNR}=30.4 \mathrm{~dB}$ and $\mathrm{NMSE}=1.34 \%$ and NASE $=5.36 \%$ for $\mathrm{SNR}=40.3 \mathrm{~dB}$. Figures 10 and 11 illustrate the results.

\section{Discussion}

The inversion of the CRT transform by the filtered back-projection algorithm involves the action of the filter $\frac{u^{2}+v^{2}}{\cos ^{3} \omega}$. For fixed $\omega$, this filter boosts high frequencies in the re- 

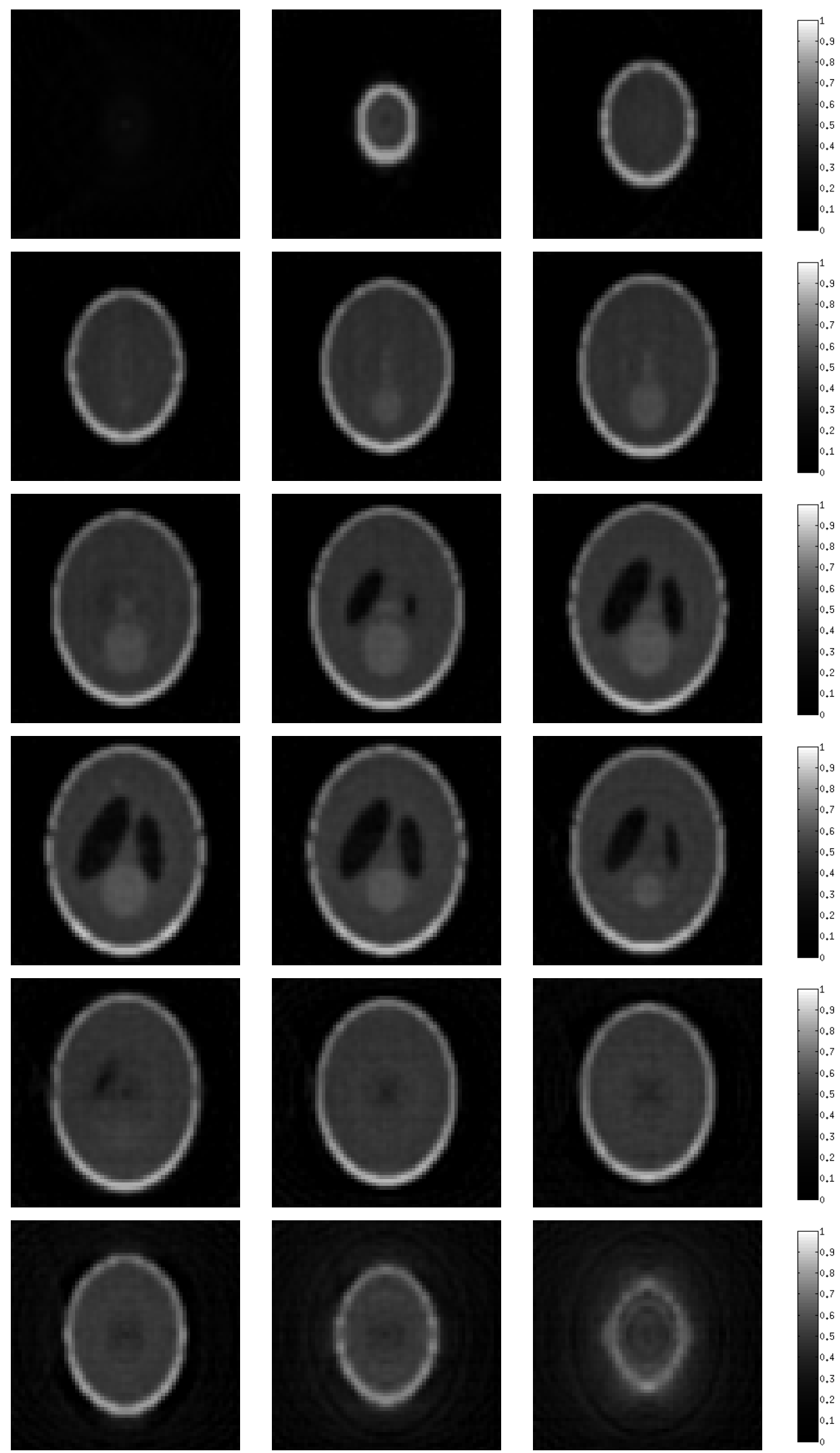

Figure 9. Reconstruction with cosine smoothing window $(\mathrm{NMSE}=1.33 \%$ and $\mathrm{NASE}=5.11 \%$ ). The same layout as in Figure 5. 

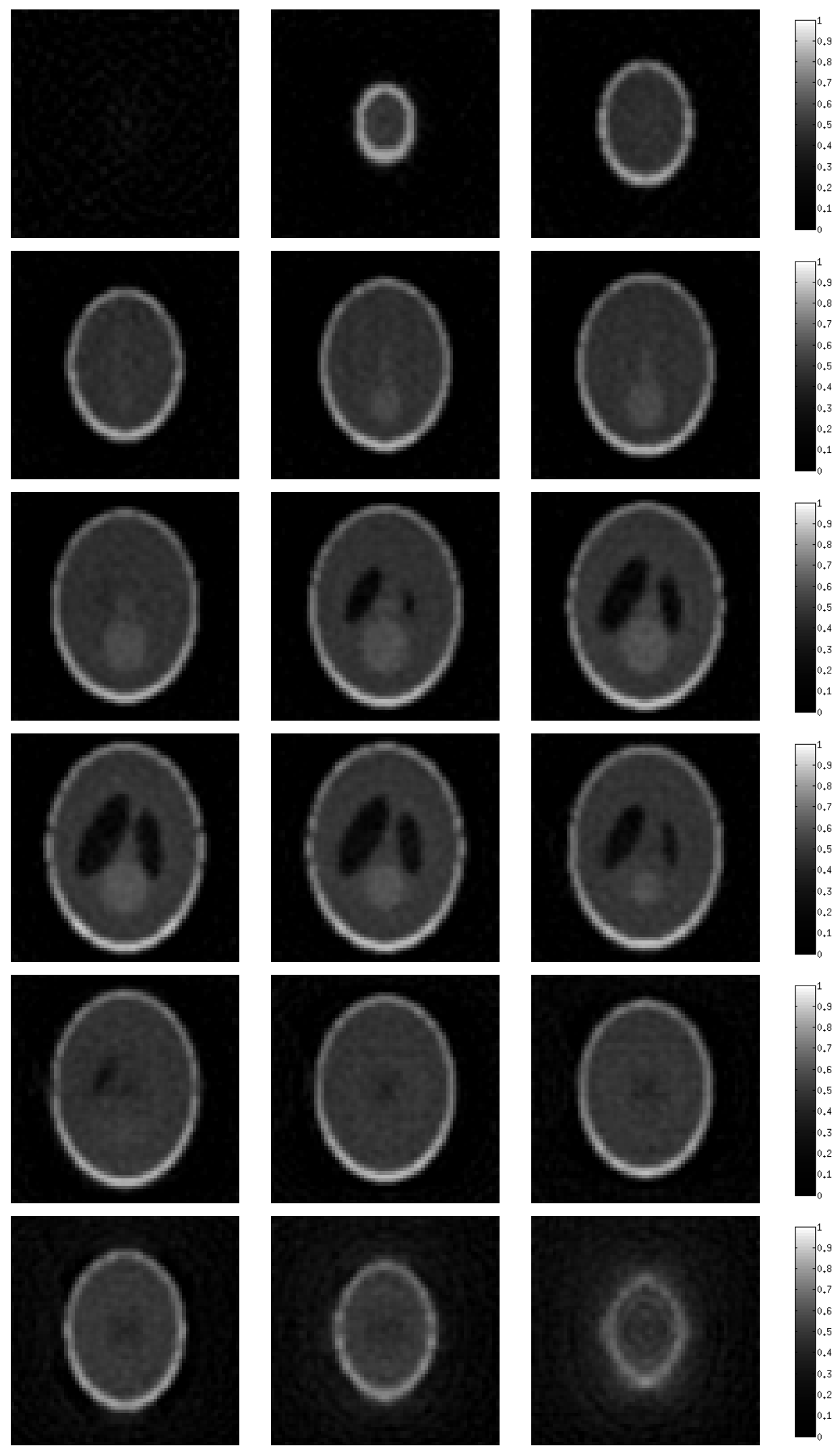

Figure 10. Reconstruction with cosine smoothing window and white gaussian noise SNR $=40 \mathrm{~dB}(\mathrm{NMSE}=1.34 \%$ and NASE $=5.36 \%$ ). The same layout as in Figure 5 . 

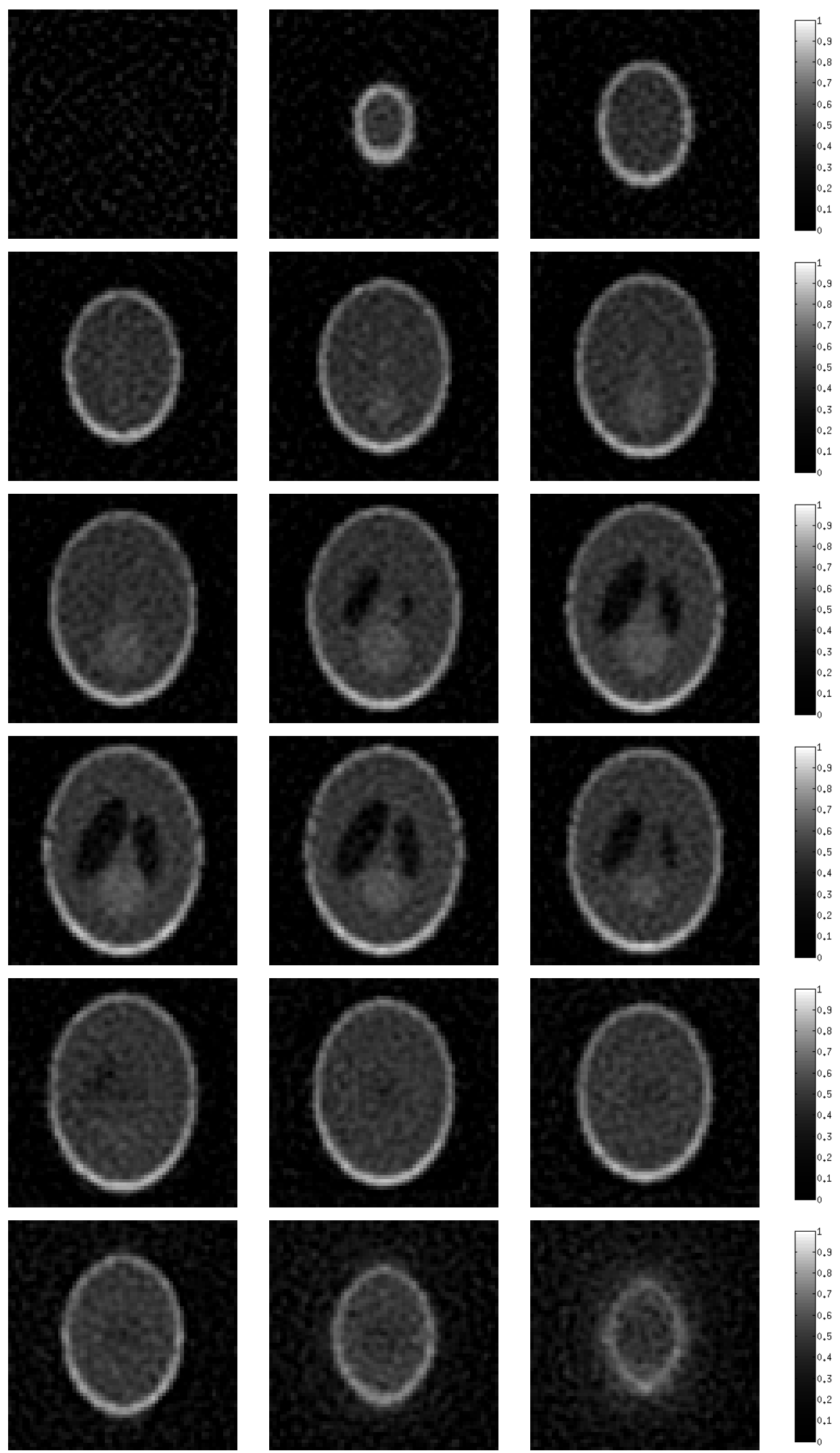

Figure 11. Reconstruction with cosine smoothing window and white gaussian noise $\mathrm{SNR}=30 \mathrm{~dB}$ (NMSE $=1.46 \%$ and NASE $=6.39 \%$ ). The same layout as in Figure 5 . 
construction, so a convolution with different smoothing windows (cosine, Shepp Logan or Hamming) is performed. Reconstructions from noiseless projections with apodization exhibit a general good quality. The bounds of the object are well defined, the small structures inside are properly recovered and the contrast between different zones is conserved. In what concerns figures of merit, Table 1 suggests that there is no significant difference among the smoothing windows. Figure 10 indicates that the results are acceptable for very low levels of noise. Nevertheless, for higher levels of noise (Figure 11), a denoising technique should be applied beforehand in order to obtain appropriate data for reconstruction. Regarding the reconstruction of the different slices of the phantom, the error in upper planes tends to be larger. This effect is explained by the fact that, for a given angle, photons emitted in the upper part of the medium arrive at the external parts of the detector that were not considered in the discretization (see the explanation of the truncation in Section 5.1). This problem could be addressed by expanding the planar detector.

\section{Conclusion and perspectives}

In this work we have shown that the concept of back-projection which has emerged from the study of the classical Radon transform can be applied to Radon transforms on a special class of circular cone surfaces. By means of appropriate filtering, this procedure leads to an inversion formula which is identical to the one derived analytically. We have illustrated this fact by constructing efficient reconstruction algorithms dictated by this approach and have shown its image quality on numerical simulations. This result suggests to test this procedure on other emission imaging such as [23], for which both exact and numerical results are known and propose further extensions in more complicated imaging conical Radon transforms which do not have exact analytic inverses.

\section{Acknowledgement}

The authors would like to express their deepest gratitude to Professor Tuong T. Truong for his constant support and stimulating discussions, and acknowledge support from ETIS (ENSEA/Univerity of Cergy-Pontoise/CNRS UMR 8051) for financial funding of a visit to ETIS in July 2014 of Javier Cebeiro.

\section{Funding}

Javier Cebeiro research work is supported by a grant from Consejo Nacional de Investigaciones Científicas y Técnicas (CONICET, Res. D N 3655 del 07 de diciembre de 2012).

\section{References}

[1] Todd RW, Nightingale JM, Everett DB. A proposed gamma camera. Nature. 1974:251; 132 134.

[2] Singh M. An electronically collimated gamma camera for single photon emission computed tomography: part I. Theoretical considerations and design criteria. Med. Phys. 1983:10; $421-427$. 
[3] Nguyen MK, Truong TT, Driol C, Zaidi H. On a novel approach to Compton scattered emission imaging. IEEE Trans. Nucl. Sci. 2009:56; 1430-1437.

[4] Basko R, Zeng GL, Gullberg GT. Applications of spherical harmonics to image reconstruction for the Compton camera. Phys. Med. Biol. 1998:43; 887-894.

[5] Parra L. Reconstruction of cone-beam projections from Compton scattered data. IEEE Trans. Nucl. Sci. 2000:47; 1543-1550.

[6] Truong TT, Nguyen MK, Zaidi H. The mathematical foundations of 3D Compton scatter emission imaging. International Journal of Biomedical Imaging. 2007. Article ID 92780.

[7] Maxim V, Frandes M, Prost R. Analytic inversion of the Compton transform using the full set of available projections. Inverse Problems. 2009:25. 095001.

[8] Cree MJ, Bones PJ. Towards direct reconstruction from a gamma camera based on Compton scattering. IEEE Trans. Med. Imaging. 1994:13; 398-407.

[9] Nguyen MK, Truong TT, Grangeat P. Radon transforms on a class of cones with fixed axis direction. J. Phys. A: Math. Gen. 2005:38; 8003-8015.

[10] Haltmeier M. Exact recontruction formulas for a Radon transform over cones. Inverse Problems. 2014:30. 035001.

[11] Morvidone M, Nguyen MK, Truong TT, Zaidi H. On the V-line Radon transform and its imaging applications. International Journal of Biomedical Imaging. Vol. 2010. Article ID 208179. 6 pages. 2010.

[12] Bolozdynya A. I., Ordonnez C. E. and Chang W., A concept of cylindrical Compton camera for SPECT. Nuclear Science Symposium Proceedings, Albuquerqe NM USA. 1997:2; 10471051.

[13] Nguyen MK, Truong TT. On an integral transform and its inverse in nuclear imaging. Inverse Problems. 2002:18; 265-277.

[14] Nguyen MK, Truong TT, Bui HD, Delarbre J-L. A novel inverse problem in gamma-rays emission imaging, Inverse Problems in Science and Engineering. 2004:12; 225-246.

[15] Truong TT, Nguyen MK. On a class of generalized Radon transforms and its application to imaging science. International Journal of Pure and Applied Mathematics. 2008: 49; 373-379.

[16] Magnus W, Oberhettinger F, Tricomi FG, 1981 Higher Transcendental Functions II (R E Krieger Publishing Co Malabar Florida).

[17] Lebedev NN, 1972 Special Functions and their Applications, (Dover Publications New York).

[18] Ramm AG. Inversion formula and singularities of the solution for the back-projection operator in tomography, Proc. Amer. Math. Soc. 1996:124(2); 567-577.

[19] Barrett HH. The Radon Transform and its Applications. Progress in Optics. Ed. E. Wolf, North Holland. 1984:21; 219-286.

[20] Cnops J. The relation between the dual and the adjoint Radon transforms. Proceddings of the Winter School 'Geometry and Physics', Circolo Matematico di Palermo, Palermo 1991. J. Bureš and V. Souček Eds. Rendiconti del Circolo Matematico di Palermo, Serie II, Supplemento No. 1991:26; 135-142.

[21] Hungerbühler N. Singular filters for the Radon backprojection. Journal of Applied Analysis. 1998:5(1); 17-33.

[22] Bracewell RN. Numerical Transforms. Science. 1990: 248; 697-704.

[23] Rigaud G, Nguyen MK, Louis AK. Novel Numerical Inversions of two Circular-Arc Radon Transforms in Compton scattering tomography. Inverse Problems in Science and Engineering. 2012:20; 809-839. 